\title{
Contenido mental y el cuerpo representado en la acción
}

\author{
Luis Murillo Jara \\ Universidad Agustiniana
}

Resumen: La existencia de representaciones del cuerpo, en especial aquellas que se requeririan para actuar, ha sido objeto de largas controversias. El objetivo de este artículo es defender la idea de que hay representaciones del cuerpo involucradas en la realización de acciones cotidianas. Nuestra tesis será que lo que realmente muestran algunas dudas sobre este tipo de representaciones es que deben tener cierto tipo de contenido no-conceptual. Primero, abordamos dichas dudas, mostrando que no refutan la existencia de las representaciones en cuestión. Sin embargo, señalamos que contienen dos intuiciones clave para cualquier caracterización del contenido de dichas representaciones. Así, pasamos a proponer una caracterización tal, que acomode adecuadamente las intuiciones señaladas. Finalizamos examinando una posible objeción a la propuesta y ofreciendo una lectura tentativa de la relación entre contenidos mentales y acción.

Palabras clave: representación del cuerpo; contenido mental no-conceptual; acción; especificación del contenido mental

\begin{abstract}
Mental Content and the Body as Represented in Action". The existence of representations of the body, especially those that would be required to act, has been the subject of long controversies. The objective of this article is to defend the idea that there are representations of the body involved in carrying out daily actions. Our thesis is that what some doubts really show about this type of representation is that they must have a certain type of non-conceptual content. First, we address these doubts, showing that they do not refute the existence of the representations in question. We note that they contain two insights for any characterization of the content of such representations. Thus, we come to propose a characterization such that it adequately accommodates the indicated intuitions. We conclude by examining a possible objection to the proposal and offering a tentative reading of the relationship between mental content and action.
\end{abstract}

Keywords: representation of the body; non-conceptual content: action; specification of mental content 


\section{Introducción}

¿Se requieren representaciones corporales para realizar acciones tan cotidianas como agarrar una taza de café o montar en bicicleta? La respuesta afirmativa de buena parte de la ciencia cognitiva y la filosofia de la mente ha suscitado interminables debates debido a críticas procedentes principalmente de la fenomenología. El objetivo de este trabajo será argumentar que llevar a cabo acciones cotidianas involucra representaciones del cuerpo cuyo contenido ha de ser de cierta clase. Sostendremos que solo si se afirma ese tipo de contenido será posible hacer frente a las diversas críticas que esta postura ha recibido.

Empezaremos presentando y discutiendo dos críticas de Merleau-Ponty a la idea de que tanto la cognición del cuerpo como la realización de acciones son representacionales, procurando establecer su alcance y precisar sus supuestos. Después, resaltaremos dos implicaciones de estas críticas, señalando que deben ser tenidas en cuenta por cualquier explicación de las representaciones del cuerpo involucradas en la orientación de acciones cotidianas. A continuación, presentaremos una propuesta de caracterización de este tipo de representaciones, que se atiende adecuadamente tales consideraciones. Alli destacaremos las principales consecuencias de la propuesta, en particular las que se relacionan con la distinción entre actitud y contenido y las que tienen que ver con semejanzas y diferencias con otras aproximaciones. Posteriormente, examinaremos una objeción potencial contra la manera en que sugerimos especificar el tipo de contenido de las representaciones en cuestión. Nuestra respuesta enfatizará rasgos esenciales de ciertas especificaciones que no se pueden perder de vista. Por último, haremos algunas observaciones sobre la manera en que diversos tipos de contenido mental se relacionan con la acción y el papel que allí jugaría el contenido de las representaciones del cuerpo involucradas en la orientación de acciones cotidianas.

2. Primera parte: algunas dudas sobre la existencia de representaciones del cuerpo

Una de las explicaciones más conocidas de la forma en que sabemos de nuestro cuerpo es la que propone la ciencia cognitiva, que postula la existencia de representaciones del cuerpo. Así sucede, por ejemplo, cuando se plantea la 
distinción entre el "esquema corporal" (una representación de tipo sensorimotor de propiedades espaciales del cuerpo, que se usaría para planear y controlar la acción) y la "imagen corporal" (una representación del cuerpo que se usaría para juicios) ${ }^{1}$.

Pese a lo anterior, la idea de que existen representaciones del cuerpo ha recibido numerosas críticas a lo largo de los años, en particular la tesis según la cual estas representaciones son fundamentales para orientar las acciones cotidianas. Teóricos como Gallagher ${ }^{2}$ y Dreyfus ${ }^{3}$ han argumentado que la noción de representación del cuerpo no captura la manera distintiva en la que sabemos de él. En su opinión, nuestras acciones cotidianas no involucran representaciones del cuerpo y, más aun, una noción como la de representación del cuerpo convierte la relación entre el cuerpo y la acción en algo innecesariamente mediado e indirecto (debilitando el papel del cuerpo mismo en una explicación de la acción).

Buena parte de los argumentos de estos teóricos toman su inspiración del trabajo de Merleau-Ponty. De acuerdo con este autor ${ }^{4}$, la única forma en que el cuerpo podría ser percibido sería como un objeto fisico cualquiera ${ }^{5}$. Sin embargo, continúa, experimentamos nuestro cuerpo como aquello que posibilita la percepción, lo cual es incompatible con que sea para nosotros un objeto físico como los demás. Así, la forma en que experimentamos nuestro cuerpo no puede hacer parte de una percepción. Adicionalmente, afirma Merleau-Ponty ${ }^{6}$, la idea de que el cuerpo puede ser percibido conduce a una regresión infinita: si el cuerpo es dado como lo que posibilita la percepción y si el objeto intencional de la percepción es dado como externo, entonces percibir el cuerpo requeriría otro cuerpo que posibilitara esta percepción y así sucesivamente. La manera más efectiva (y menos arbitraria) de evitar esta regresión infinita parecería ser negando que el cuerpo, en tanto que nos es dado como lo que posibilita la percepción, pueda ser percibido.

1 Cf., De Vignemont, F., "Body Schema and Body Image-Pros and Cons", en: Neuropsychologia, v. XLVIII, 3 (2010), pp. 669-680.

2 Gallagher, S., How the Body Shapes the Mind. Nueva York: Oxford University Press, 2005.

3 Dreyfus, H., "Intelligence without representation - Merleau-ponty's Critique of Mental Representation the Relevance of Phenomenology to Scientific Explanation", en: Phenomenology and the Cognitive Sciences, v. IV, 1 (2002), pp. 367-383.

4 Merleau-Ponty, M., Phénoménologie de la Perception, París: Gallimard, 1945, p. 108.

5 La argumentación de Merleau-Ponty en contra de la idea según la cual el cuerpo es objeto de percepción se puede extender igualmente a la idea de que el cuerpo es objeto de representación (cf., Merleau-Ponty, Phénoménologie de la Perception, p. 109). En este sentido, asumiremos que dicha argumentación se aplica por igual a esas dos ideas.

6 Ibid., p. 107. 
El rechazo de Merleau-Ponty a la idea de que el cuerpo puede ser percibido (o representado) depende de la afirmación de que, si así fuera, el cuerpo sería dado como un objeto físico cualquiera. Lo que parece estar detrás de esta afirmación es la siguiente suposición: que el objeto intencional de la percepción (o las representaciones) es necesariamente dado como externo y determinado. Sin embargo, no hay razones para pensar que esta sea una suposición natural o forzosa. El contenido de una percepción o de una representación podría no ser algo completamente determinado (por ejemplo, a causa de algunas circunstancias perceptuales o de nuestro conocimiento previo) o podría no ser dado como externo (por ejemplo, si se trata de un objeto mental o de un objeto abstracto, entre otras). Si rechazamos la mencionada suposición, que el cuerpo fuera representado no significaría que por ello fuera dado como un objeto físico cualquiera (abriendo así la posibilidad de que sea representado como aquello que hace posible la percepción).

Como alternativa a la noción de representación del cuerpo, Merleau-Ponty propone reconocer la existencia de una forma de intencionalidad que se localiza entre la que denomina intencionalidad "de acto" ("la de nuestros juicios", es decir, la intencionalidad tética o tematizante, explícita) y las repuestas motoras puramente mecánicas o reflejas 7 . La intencionalidad "motora"8 u "operante"9, descrita como antepredicativa, prerreflexiva y motriz, daría cuenta de las formas de interacción corporal en las que cotidianamente nos involucramos -que no son resultado de un plan deliberado y explicito, ni meras respuestas motoras automáticas o reflejas-. No sería, entonces, la intencionalidad de acto, ni los modelos de estímulo-respuesta, sino la intencionalidad motora u operante lo que explicaría nuestras acciones cotidianas ${ }^{10}$.

Sin embargo, no es claro que los conceptos de intencionalidad motora u operante y de contenido representacional sean excluyentes. Pensemos en el tipo de ejemplo que prefieren Merleau-Ponty y sus partidarios: la manera en que preparamos nuestro cuerpo para agarrar un objeto. Esto lo hacemos a partir de una manera en la que nos es dado dicho objeto. Así, si podemos decir que nuestra disposición corporal es guiada por la forma en la que nos son dados nuestros alrededores, podemos decir que es guiada por contenido mental en el

\footnotetext{
Merleau-Ponty, M., Phénoménologie de la Perception, p. 128.

Ibid.

Ibid., p. 478.

${ }^{10}$ Arias, B., "La intencionalidad operante en Maurice Merleau-Ponty", en: Contrastes: revista internacional de filosofia, v. II (1997), pp. 5-25.
}

ARETÉ Revista de Filosofía, v. XXXII, 1, 2020 / ISSN 1016-913X 
sentido de Cussins ${ }^{11}$. Esto no implica, sin duda, que se requiera tipo alguno de reflexión para que nuestro entorno nos sea presentado.

\subsection{El alcance de las dudas sobre las representaciones del cuerpo}

Si los anteriores argumentos para rechazar las representaciones corporales no son concluyentes, y si el concepto de intencionalidad motora u operante no excluye el de contenido representacional a la hora de explicar la manera en que son orientadas nuestras acciones cotidianas, podría pensarse que el representacionalismo ha salido incólume en este asalto. Ahora bien, este no es el caso. Como se mostrará a continuación, dos poderosas intuiciones estarían detrás del rechazo de Merleau-Ponty a la noción de representación del cuerpo y en su noción de intencionalidad motora u operante. Como se argumentará a continuación, estas dos intuiciones, de hecho, condicionan cualquier posible explicación del contenido de las representaciones del cuerpo que orientan nuestras acciones cotidianas.

La primera intuición se asocia con la idea de que nuestro cuerpo no nos es dado como un objeto físico cualquiera. Por supuesto, nuestro cuerpo es un objeto físico que se rige por las mismas leyes que rigen a los demás objetos físico y que interactúa causalmente de la misma manera que estos. Pensemos, por ejemplo, en la ley de inercia: si vamos en un vehículo en movimiento y este gira, nuestro cuerpo tenderá a conservar la dirección que llevaba antes del giro. Esto mismo sucede con la velocidad de caída libre, etcétera. De manera semejante, al igual que otros objetos físicos, nuestro cuerpo tiene ciertas propiedades a las que accedemos por medio de experiencias perceptuales (como color, textura y forma). A pesar de ello, para nosotros nuestro cuerpo es radicalmente distinto de los demás objetos físicos.

Consideremos ciertos rasgos de la manera en que sabemos de nuestro cuerpo que nos son tan familiares que a veces los pasamos por alto. Estos rasgos son de crucial importancia para evidenciar la manera singular en que nuestro cuerpo nos es dado. En primer lugar, como observa Merleau-Ponty, nuestro cuerpo siempre nos es dado "bajo el mismo ángulo"; de allí que resulte extraña la idea de cambiar nuestra posición con respecto a nuestro cuerpo. Asimismo, a diferencia del lugar donde se ubican los demás objetos, experimentamos el lugar donde se ubica nuestro cuerpo como el punto hacia al que se orientan

11 Cussins, A., "Content, Conceptual Content, and Nonconceptual Content", en: Essays on Nonconceptual Content, MIT Press, 2002, pp. 133-163. 
nuestras percepciones (visuales, auditivas). Cuando observamos o escuchamos el mundo circundante, nuestras percepciones contienen una perspectiva cuyo origen es la región del espacio en la que se encuentra ubicado nuestro cuerpo ${ }^{12}$.

Otra de las características que hacen particular nuestra experiencia de nuestro cuerpo es que podemos saber cuál es su postura general y en qué posición se encuentran sus partes sin requerir experiencias visuales, táctiles o, en general, sin usar las típicas modalidades sensoriales. Además, no solo recibimos información abundante, inmediata y permanente sobre el estado de nuestro cuerpo, sino que parte de ella es de un tipo único: acerca del estado interno de nuestro cuerpo, algo que dificilmente podemos saber de los demás objetos. Con todo, existe un rasgo de la manera en la que sabemos de nuestro cuerpo que evidencia de manera más dramática que este nos es dado de una manera diferente a como nos son dados los objetos a nuestro alrededor: solo este objeto es tal que sus movimientos son resultado directo o inmediato de nuestra voluntad. Cualquier otro objeto que deseemos mover debemos moverlo con nuestro cuerpo, pero para mover nuestro cuerpo no necesitamos ningún intermediario. Como señala O’Shaughnessy ${ }^{13}$, nuestro cuerpo es el objeto inmediato de la voluntad.

Lo anterior muestra que, si bien es un objeto, nuestro cuerpo no nos es dado como cualquier otro objeto físico. En consecuencia, una teoría de la manera en que representamos objetos no necesariamente será capaz de dar cuenta de la manera en que representamos nuestro cuerpo. Si dicha teoría es incapaz de incorporar las diferencias profundas entre la manera en la que nuestro cuerpo nos es dado y la manera en la que los demás objetos nos son dados, resultará inadecuada para dar cuenta del contenido de las representa-

\footnotetext{
${ }^{12}$ Vale la pena señalar aquí que algunos casos neuropsicológicos parecen sugerir que este aspecto de la manera en la que sabemos de nuestro cuerpo puede ser contingente. Algunos sujetos reportan la experiencia de ver su cuerpo desde un punto exterior al mismo, junto a la sensación de estar fuera de su cuerpo y experimentando que su cuerpo está en el espacio extrapersonal. De acuerdo con Blanke y Mohr la autoscopia puede deberse a que el procesamiento multisensorial presenta una desintegración funcional, y también a que en el área temporoparietal se da un procesamiento anormal ( $c f$. "Out-of-Body Experience, Heautoscopy, and Autoscopic Hallucination of Neurological Origin: Implications for Neurocognitive Mechanisms of Corporeal Awareness and Self-consciousness", en: Brain Research Reviews, v. I, 50 (2005)). Sin embargo, más allá del sustrato cerebral de este fenómeno, no es claro cómo deben entenderse algunas de sus características: “¿cómo me puede ser dado como propio un cuerpo que veo como externo?”, “¿cómo me es dado el punto de vista desde el que observo mi cuerpo?” En este sentido, no es suficientemente claro cómo deberian describirse las experiencias de los sujetos con autoscopia: si se trata más bien de un "tengo la experiencia visual de un cuerpo que se ve justo como mi cuerpo" o "experimento como mi propio cuerpo este cuerpo que veo".

13 O’Shaughnessy, B., The Will, v. I, Cambridge: Cambridge University Press, 1980, p. 267.
} 
ciones del cuerpo (incluidas aquellas involucradas en la orientación de nuestras acciones cotidianas) ${ }^{14}$.

La segunda intuición, que condicionaría cualquier explicación del contenido de las representaciones del cuerpo involucradas en la orientación de la acción cotidiana, se relaciona con algunas características de la intencionalidad motora/operante de Merleau-Ponty. El contenido de estas representaciones debe estar conectado con la acción, siendo suficiente para que esta tenga lugar, si ha de explicar las acciones cotidianas. De lo contrario, se correría el riesgo de dejar brechas o algún tipo de armonía preestablecida entre este contenido y la acción.

Precisamente en este orden de ideas es que Merleau-Ponty señala que "queda por entender por qué operación mágica la representación de un movimiento suscita justamente en el cuerpo ese mismo movimiento"15. El término "mágica" parece referirse a la falta de claridad sobre la conexión entre estas representaciones y la acción corporal. Por ejemplo, no es claro que una creencia implique acciones corporales, por lo cual habría que decir cómo es posible que las produzca. Al respecto, Alsmith y de Vignemont ${ }^{16}$ anotan que las creencias también están involucradas en la orientación del pensamiento abstracto y que "como tal, [la creencia] no está intrínsecamente centrada-en-la-acción... no es suficiente para desencadenar su acción"17.

Más aun, las creencias no implicarian acciones incluso cuando se trata de creencias que indican que conviene realizar tal o cual acción. Pensemos en alguien que cree que debería pedirle un aumento de salario a su jefe o en alguien que cree que debería invitar a una cita a otra persona. Como lo muestra la experiencia, estas creencias no necesariamente incitan a los agentes a llevar a cabo las acciones (básicas y no-básicas) correspondientes ${ }^{18}$.

\footnotetext{
${ }^{14}$ En todo caso, siguiendo a O'Shaughnessy, B., del hecho de que experimentemos nuestro cuerpo como algo distinto de cualquier otro objeto fisico no se sigue que no puede haber percepción o representación del mismo ( $c f$. "Proprioception and the Body Image", en: The Body and the Self, MIT Press, 1995).

15 Merleau-Ponty, M., La Phénomenologie de la Perception, p. 63. La traducción es mía.

16 Alsmith, A. y F. de Vignemont, "Embodying the mind and representing the body", en: Review of Philosophy and Psychology, v. I, 3 (2012), pp. 1-13, p. 6

${ }_{17}$ Una observación similar es hecha por Millikan, quien afirma que las representaciones puramente descriptivas no sirven para guiar la acción ( $c f$. Millikan, R., "Pushmi-pullyu Representations", en: Philosophical Perspectives, v. IX (1995), p. 192). Para incidir en la acción, estas representaciones deben estar combinadas con representaciones directivas.

${ }^{18}$ Esto recuerda el ataque de Searle al "modelo clásico", según el cual las razones causan nuestras acciones. Como objeta Searle, contar con razones suficientes para realizar una acción -entre las que pueden encontrarse creencias- no siempre causa que un sujeto actúe en consecuencia (incluidas las acciones básicas correspondientes) (cf. Searle, J., Rationality in action, Cambridge:
} 


\subsection{Contenido conceptual, contenido no-conceptual y acción}

A lo anterior se podria responder que, intuitivamente, tener creencias es una condición necesaria para actuar (¿cómo podría tratar de abrir la puerta si no creyera que está cerrada?). No habría brecha alguna, pues las creencias y otras actitudes proposicionales parecen incidir directamente sobre las acciones correspondientes. Sin embargo, la idea de que las creencias son condición necesaria para la acción es discutible. Piénsese en una persona que va en su bicicleta: evidentemente, puede que lo haga con la intención de ir a algún destino, pero, al hacerlo, pedalea con cierta frecuencia, gira con inclinaciones específicas, etcétera. Siguiendo la intuición mencionada al comienzo del párrafo, se podría decir que el ciclista sabe que si pedalea con esa frecuencia alcanzará una velocidad específica, o que cree que al girar su cuerpo con esa inclinación específica evitará caerse (y que, además, no desea caer). Tal intuición colapsa al notar que esos estados mentales rara vez hacen parte de nuestra experiencia de ir en bicicleta. Sin duda, necesito pedalear con cierta frecuencia para alcanzar cierta velocidad y necesito girar con cierta inclinación si no he de caer. Del mismo modo, puede decirse que experimento de cierta manera la velocidad a la que voy y la inclinación con la que giro. Sin embargo, no necesitamos tener creencias acerca de velocidad e inclinación para ir en bicicleta. De hecho, un sujeto puede tener creencias sobre la frecuencia con la que hay que pedalear y la inclinación con la que hay que girar para ser un hábil ciclista y, aun así, ser incapaz de montar una bicicleta. Así, las creencias y otras actitudes proposicionales no solo no serían condición necesaria, sino que tampoco serían condición suficiente para realizar ciertas acciones.

Quizás los deseos sean mejores candidatos a actitudes proposicionales capaces de producir directamente una acción corporal, pues parecen contener un aspecto motivacional. Sin embargo, no es gratuito que las teorías sobre actitudes proposicionales prefieran concentrarse en las creencias más que en los deseos (lo que Lycan llama "la abrumadora preeminencia social sobre las demás actitudes"19). Para empezar, no es obvio que los deseos sean actitudes

122 proposicionales, pues no es claro si solo pueden ser acerca de estados de cosas

\footnotetext{
MIT press, 2001). En otras palabras, Searle sostiene que las "intenciones previas" pueden dar lugar a acciones, pero que no necesariamente lo hacen. Según este autor, cuando las intenciones previas producen acciones es gracias a que están relacionadas con "intenciones-en-la-acción", las cuales controlan y guían la conducta directamente.

${ }^{19}$ Lycan, W., "Desire Considered as a Propositional Attitude", en: Philosophical Perspectives, v. XXVI, 1 (2012), pp. 201-215, p. 213.
} 
o si también pueden ser acerca de objetos. Solo en el primer caso tendrian necesariamente contenido proposicional, pero el sentido común parece admitir que algunos deseos son acerca de objetos ${ }^{20}$. Adicionalmente, Lycan ha mostrado que la explicación de los deseos en términos de actitudes proposicionales deja por fuera características importantes de la satisfacción de deseos. Como señala este autor, cuando se trata de los deseos "hay un problema serio con respecto a la naturaleza de su contenido"21. Aun más, la conexión de los deseos con la acción podría ser contingente: Strawson ${ }^{22}$ ha sostenido que se pueden concebir criaturas que carecen de disposiciones a actuar y que, aun asi, tienen deseos (los cuales consistirian en disposiciones a sentir placer y displacer).

Que las actitudes proposicionales no produzcan directamente acciones corporales, por supuesto, no significa que sean irrelevantes para la explicación de nuestras acciones. En muchos casos resultan clave para racionalizarlas la acción. No obstante, se requiere algo que permita conectar su contenido proposicional con la acción.

Cussins $^{23}$ sostiene que únicamente si es intrínsecamente motivacional un contenido mental podrá ser capaz de producir acciones directamente. De acuerdo con este autor, esta conexión con la acción debe describirse capturando el modo intrínsecamente motivacional en el que el ambiente le es presentado al sujeto. Sin embargo, esta no es la forma en la que se entiende el contenido de las actitudes proposicionales. Así, para que el contenido de las representaciones del cuerpo involucradas en la orientación de nuestras acciones cotidianas sea condición suficiente para dichas acciones, debe ser un contenido intrínsicamente motivacional. Como anotan Alsmith y de Vignemont, debe tratarse de un contenido "directamente explotable para la acción"24.

En resumen, acciones como montar en bicicleta y cruzar la calle no son automáticas, sino que son orientadas por contenidos mentales. Sin embargo, se sigue de la segunda intuición extraída de la postura de Merleau-Ponty que las representaciones del cuerpo involucradas en la orientación de nuestras acciones cotidianas tienen un contenido distinto del de las actitudes proposicionales

\footnotetext{
${ }^{20}$ Cf., Brewer, T., "Three Dogmas of Desire", en: Values and Virtues: Aristotelianism in Contemporary Ethics, Oxford: Oxford University Press, 2006, pp. 257-84 y Thagard, P., "Desires are not propositional attitudes", en: Dialogue, v. I, 45 (2006), pp. 151-156.

21 Lycan, W., "Desire Considered as a Propositional Attitude".

22 Strawson, G., Mental Reality, Cambridge: MIT Press, 1994.

${ }^{23}$ Cussins, A., "Environmental Representation of the Body", en: Review of Philosophy and Psychology, v. III, 1 (2012), pp. 15-32.

24 Ibid.
} 
(pues este no es capaz de producir acciones corporales y no está conectado de manera directa con dichas acciones) ${ }^{25}$. De contenidos proposicionales no se derivan acciones corporales, sino a lo sumo más contenidos proposicionales. En palabras de Cussins ${ }^{26}$, los contenidos proposicionales y sus componentes conceptuales son "hacedores de verdad" (truth-makers), mientras que lo que requerimos en "hacedores de acciones". Por ende, más que proposicional o conceptual, el contenido de las representaciones del cuerpo involucradas en la orientación de nuestras acciones cotidianas debe ser de tipo no-conceptual.

Dicho contenido no-conceptual orientado a la acción deberá estar constituido de tal modo que, al mismo tiempo, produzca una tendencia a actuar en el sujeto y sea posea intencionalidad. ¿Cómo cumplir esta doble función? Una suposición natural es que este contenido le presenta al sujeto elementos del ambiente al tiempo que le produce una inclinación a actuar si tal presentación es en términos de acciones concretas que el sujeto puede realizar (esto es, en términos de posibilidades de acción). Así como es una fruta lo que es presentado como comestible o un espacio lo que es presentado como accesible, etcétera, puede decirse que estas posibilidades de acción contienen también una referencia a elementos del entorno.

De este modo, el contenido en cuestión podría ser explotado de manera directa para orientar las acciones cotidianas, por lo cual no se requeriría que mediaran procesos de deliberación o reflexión que las produjeran. Las presentaciones en términos de posibilidades de acción que orientan nuestras acciones de manera directa e inmediata (sin requerir procesos inferenciales o conocimiento conceptual) permiten explicar que no debamos formar planes o tener intenciones previas cada vez que actuamos ${ }^{27}$.

${ }^{25}$ El contenido de una actitud proposicional es una proposición en la cual se articulan conceptos. En esta medida, dicho contenido es denominado "proposicional" o "conceptual" (cf., Crane, T., "Conceptual and Non-conceptual Content", en: The Routledge Encyclopedia of Philosophy, Taylor and Francis, Londres: Routledge, 1998).

26 Cussins, A., "Content, Conceptual Content, and Nonconceptual Content", p. 150.

27 La conceptualización teórica más difundida sobre aprehensión directa e inmediata de posibilidades de acción se encuentra en la teoría ecológica de la percepción de Gibson, específicamente, en el concepto de "affordance". Para el autor, la affordances son oportunidades para actuar especificadas en la información visual (contenida, a su vez, en la luz ambiental) que resultan de, por un lado, la relación entre las propiedades fisicas y habilidades del animal y, por otro lado, de las propiedades físicas del ambiente circundante ( $c f$. Gibson, J., The Ecological Approach to Visual Perception, Boston: Houghton Mifflin, 1979). Sin embargo, aunque está estructurado en términos de posibilidades de acción, el contenido que nos ocupa tiene diferencias cruciales con las affordances. En particular, hay que recordar que Gibson propuso el concepto de affordance como alternativa al de representación mental (pese a que admitía que las affordances le proporcionan al animal información sobre su ambiente y sobre sí mismo), enfatizando que las leyes de la "óptica 


\subsection{Implicaciones de la propuesta}

Ciertas consecuencias se derivan de esta concepción del contenido de las representaciones del cuerpo involucradas en la orientación de acciones cotidianas. Para empezar, Clark ${ }^{28}$ y Wheeler $^{29}$ han subrayado que cuando un contenido representacional tiene la estructura de una posibilidad de acción, es decir, cuando su modo de presentación son acciones concretas que el sujeto puede realizar, al sujeto no le es dado sino aquello que es relevante a la luz del contexto y los requerimientos de la actividad que está llevando a cabo. Por ende, solo le son dados parcialmente aquellos elementos del entorno con los que interactúa, esto es, los objetos no se le presentan como particulares re-identificables con propiedades independientes del contexto, sino como elementos dependientes del contexto de actividad.

Con esto en mente, podemos indicar con mayor precisión por qué el contenido de las actitudes proposicionales no puede dar lugar por sí mismo a acciones corporales. Una forma de contenido representacional que se pueda explotar directamente en la acción debe ser profundamente dependiente de los contextos de actividad, lo cual supone una diferencia radical con formas de contenido representacional proposicionales o conceptuales (típicamente independientes del contexto, generales y objetivas). Dicho de otro modo, una forma de contenido directamente conectada con acciones concretas posee una dependencia del contexto que le impide ser general y objetiva, como el contenido proposicional o conceptual ${ }^{30}$. Así, una forma de contenido mental exhibirá o bien propiedades como ser general y objetivo, o bien tendrá una conexión directa con nuestras acciones. En esta medida, el contenido de las representaciones del cuerpo involucradas en la orientación de acciones cotidianas será dependiente del contexto de actividad y no podrá ser general y objetivo, es decir, no podrá ser un contenido proposicional o conceptual.

En segundo lugar, la distinción entre actitud y contenido dejaría de ser pertinente en el caso del contenido que nos ocupa. De acuerdo con esta distinción, una actitud es una relación que un sujeto tiene con un contenido

\footnotetext{
ecológica” eran suficientes para explicarlas. Por su parte, el contenido que intentamos precisar es, evidentemente, un tipo de contenido mental representacional.

${ }^{28}$ Clark, A., Being There: Putting Brain, Body and World Together Again, Cambridge: MIT Press, 1997.

${ }^{29}$ Wheeler, M., Reconstructing the Cognitive World: The Next Step, Cambridge: MIT Press, 2005.

${ }^{30}$ Cussins, A., "Content, Embodiment, and Objectivity: The Theory of Cognitive Trails", en: Mind, CDIV, v. CI (1992), pp. 651-688, p. 683.
} 
neutral ${ }^{31}$. Dado que el contenido es neutral, la actitud es aquello con lo que se captura el "modo cognitivo" de un estado mental: es decir, la diferencia en el significado cognitivo de creer que $p$, desear que $p$, etcétera.

Sin embargo, en el contenido de las presentaciones del cuerpo involucradas en la orientación de nuestras acciones cotidianas el lugar significado cognitivo es distinto, pues no es planteado como un contenido neutral. De acuerdo con lo que hemos venido planteando, cuando se caracteriza el contenido de dichas representaciones se captura también el modo de presentación que este tiene para el sujeto, esto es, su significado cognitivo. De esta manera, sin la pretensión de caracterizar el contenido por un lado y su significado cognitivo por otro lado, no parece que necesitemos la distinción entre actitud y contenido: especificar este contenido y capturar su significado para el sujeto son una y la misma cosa.

En tercer lugar, vale la pena mencionar que nuestra caracterización del contenido de las presentaciones del cuerpo involucradas en la orientación de acciones cotidianas se ajusta a la tesis de Millikan ${ }^{32}$ según la cual para determinar un contenido representacional es necesario fijarse en la manera en que es usado (por ejemplo, como en nuestro caso, la manera en que se usa para guiar la acción). Del mismo modo, puede decirse que el contenido de estas representaciones del cuerpo co-varía con aquello que representa, como Millikan sostiene que deben hacer las representaciones. Por último, este contenido podría ser descrito como mediando la producción de formas de conducta que co-varían directamente con el entorno, como son definidas las representaciones pushmipullyu $^{33}$. Millikan señala que las representaciones pushmi-pullyu tienen una función descriptiva y también una función directiva, siendo a la vez más primitivas que las representaciones exclusivamente descriptivas o exclusivamente directivas. Por ello Millikan afirma que el contenido de una representación pushmi-pullyu no equivale a la conjunción de una representación puramente directiva y una puramente descriptiva. Pese a ello, Millikan ${ }^{34}$ admite que las representaciones pushmi-pullyu sean especificadas como la conjunción de una especificación directiva y otra descriptiva. Esto marca una diferencia importante entre las representaciones pushmi-pullyu y nuestra aproximación

\footnotetext{
${ }^{31}$ Oppy, G., "Propositional Attitudes", en: The Routledge Encyclopedia of Philosophy, Taylor and Francis, 1998.

${ }^{32}$ Millikan, R., "Pushmi-pullyu Representations", en: Philosophical Perspectives, v. IX (1995), pp. 185-200.

${ }^{33}$ Ibid., p. 190.

${ }^{34}$ Ibid.
} 
al contenido de las presentaciones del cuerpo involucradas en la orientación de acciones cotidianas, pues estas últimas no "mapean" el entorno de una forma que pueda ser especificada adecuadamente mediante la conjunción de enunciados descriptivos y directivos distintos. Una especificación adecuada del contenido de estas representaciones, que reconozca el significado cognitivo que tiene para el sujeto y que lo integre con su dimensión referencial, sería en términos de posibilidades de acción.

La especificación que Millikan admite para las representaciones pushmipullyu no es adecuada para su contenido y parece renunciar al objetivo de capturar el significado cognitivo que estas representaciones tienen para sus sujetos, manteniéndose dentro de los límites de las especificaciones de creencias y deseos. Para Millikan ${ }^{35}$ las representaciones mentales tienen una función biológica propia, a saber, mapear el entorno de acuerdo con un conjunto de reglas -las cuales están fijadas por el modo en que el sistema está estructurado para interactuar con su medio ambiente-. Pese a que, en principio, este enfoque parece permitir una amplia variedad de manera en que se puede mapear el entorno (como la que hemos propuesto para el contenido que nos ocupa), Millikan no va más allá de la dicotomía entre especificaciones descriptivas y directivas.

\section{Segunda parte: los límites de la especificación del contenido}

En este apartado deseo analizar una objeción potencial para la especificación del contenido de las representaciones del cuerpo involucradas en la orientación de nuestras acciones cotidianas en términos de posibilidades de acción. Cussins ${ }^{36}$ ha argumentado que ninguna especificación que tenga condiciones de verdad puede corresponder a un contenido no-conceptual. Solo las proposiciones pueden ser falsas o verdaderas, por lo que una especificación con condiciones de verdad le estaría atribuyendo una proposición o contenido proposicional al sujeto. La conclusión de Cussins es que debe afirmarse que las especificaciones del contenido mental no-conceptual no tienen valor de verdad. La pregunta que hay que responder es cómo puede algo ser una presentación del mundo sin tener valor de verdad (pues, si el mundo es como lo presenta cierto contenido, lo que parece seguirse es que se trata de un contenido verdadero).

35 Ibid., p. 186.

${ }^{36}$ Cussins, A., "Content, Embodiment, and Objectivity: The Theory of Cognitive Trails". 
Según Cussins ${ }^{37}$, la especificación adecuada del contenido mental no-conceptual no puede contener la suposición de una distinción entre mente y mundo. De este modo, introduce un lenguaje que no tiene la estructura sujetopredicado y en el que la semántica de las oraciones solo involucra localizar rasgos demostrativamente (en lugar de identificar particulares). El significado de las oraciones de este lenguaje de localización de rasgos se limita, pues, a la alusión de la región general en la que ciertos rasgos se presentan, y la capacidad para indicar un rasgo no requiere ser capaz de identificar y reidentificar objetos o lugares. En consecuencia, continúa Cussins, cuando un contenido no-conceptual es especificado mediante oraciones de dicho lenguaje (las cuales podrian ser complementadas con oraciones ordinarias por parte del teórico), se ha capturado el modo de presentación característico que este tiene para el sujeto. Por lo demás, la particular semántica de este lenguaje no permite considerar que sus expresan una proposición, de modo que no pueden ser verdaderas ni falsas.

Con todo, varios elementos del argumento por el cual Cussins concluye que solo este tipo de especificación funcionaría para el contenido mental no-conceptual parecen controvertibles. Específicamente, Cussins podría estar confundiendo cosas distintas cuando afirma que un contenido es verdadero si el mundo es como este lo presenta ${ }^{38}$, o que si la especificación de un contenido tiene valor de verdad es un contenido conceptual ${ }^{39}$.

Para que una representación mental tenga un cierto contenido, debe cumplirse una condición usualmente denominada la "condición de verdad" de ese contenido. Este nombre viene de que el cumplimiento de esa condición (1) haga verdadera cierta proposición que especifica el contenido en cuestión y (2) permita afirmar que dicho contenido mapea el entorno de manera confiable (y, así, pueda decirse que en cierto sentido es un contenido "verdadero"). No obstante, la posibilidad misma de que haya contenidos mentales no-conceptuales depende de que no sea necesario que el sujeto conozca proposicionalmente dicha condición para poseer el contenido respectivo, de lo cual se sigue que una cosa es poseer un contenido y otra distinta es comprender una proposición que lo especifica. Asimismo, si se dice que un contenido es "verdadero" porque mapea el entorno de manera confiable, se hace un uso laxo del término. Formas de contenido mental no-conceptual como el que Peacocke ${ }^{40}$ denomina

${ }^{37}$ Ibid., p. 669.

${ }^{38}$ Ibid., p. 664

39 Ibid.

40 Peacocke, C., "Scenarios, Concepts, and Perception", en: The Contents of Experience, Cambridge University Press, 1992, pp. 105-135.

ARETÉ Revista de Filosofia, v. XXXII, 1, 2020 / ISSN 1016-913X 
"contenido de escenario" (scenario content) mostrarian que un contenido puede 'mapear' el entorno de manera confiable sin que por ello sea proposicional (ergo verdadero o falso).

Así, cuando se cumple la condición que le permite a una representación tener cierto contenido, aquello que es verdadero es su especificación, no necesariamente el contenido mismo. En suma, del hecho de que exista una condición cuyo cumplimiento significa que un contenido mental mapea el entorno de manera confiable y que la especificación del contenido correspondiente es verdadera, no se sigue que el contenido en cuestión es contenido mental conceptual. En otras palabras, que tal contenido mapee el entorno de manera confiable y que su especificación sea verdadera no implica necesariamente que lo que se le presenta al sujeto es una parte de un mundo objetivo compuesto por particulares. Es la posesión de contenido mental conceptual la que requiere que el sujeto comprenda una proposición que especifica su contenido, en la que se articulan conceptos y que presenta el mundo en términos de objetos y propiedades. De allí que pueda decirse que únicamente en el caso del contenido mental conceptual poseer un contenido es lo mismo que comprender su especificación.

Cussins admite que poseer contenido mental no-conceptual es algo independiente de comprender la manera en que se especifica ${ }^{41}$ : una aproximación teórica al contenido mental no-conceptual justamente se trata de ofrecer especificaciones de maneras de acceder cognitivamente al ambiente mediante conceptos que los sujetos no necesitar poseer. Por ello, aunque una especificación incluya conceptos y tenga valor de verdad, el contenido mismo puede seguir siendo no-conceptual ${ }^{42}$.

Con todo, se replicará que el tipo de especificación que Cussins propone está en mejores condiciones de capturar el significado cognitivo del contenido mental no-conceptual. Este tipo de especificación describiría fielmente el modo de presentación característico que allí tienen para el sujeto los elementos del entorno. Sin embargo, siempre que se especifica un contenido representacional,

${ }^{41}$ Cussins, A., "Content, Conceptual Content, and Nonconceptual Content", p. 149.

${ }^{42} \mathrm{Si}$ bien puede parecer paradójico que una aproximación teórica al contenido mental no-conceptual caracterice el modo en que un sujeto representa su entorno pese a que dicha caracterización use nociones que el sujeto no comprende, esta impresión se disipa fácilmente. Justamente, es el hecho de que use nociones que el sujeto no comprende lo que permite que una aproximación de este tipo capture el significado cognitivo que el contenido tiene para su sujeto. Lograr una caracterización del modo en que los sujetos representan su entorno exigirá, en algunos casos, sobrepasar sus recursos conceptuales. 
esto se hace de manera lingüística y acudiendo a los recursos de una teoría, por lo que el resultado será inevitablemente conceptual. Se podría preguntar, ¿no es este un impedimento de principio para capturar perfectamente el significado cognitivo del contenido mental no-conceptual?

La pregunta se puede responder de la siguiente manera: lo que hace una aproximación teórica al contenido mental no-conceptual no es atribuir a los sujetos la posesión de los conceptos que utiliza, sino aquello a lo cual tales conceptos refieren. No obstante, esto aplica exactamente de la misma manera para cualquier especificación del contenido mental no-conceptual. Sin duda, habrá especificaciones cuyos recursos conceptuales se adecúen mejor que otras al contenido mental no-conceptual, pero esto no nos compromete con la afirmación de que alguna especificación capturará perfectamente el significado cognitivo del contenido no-conceptual. Más aun, razones pragmáticas podrán hacer que el teórico prefiera algunas especificaciones por encima de otras. Por consiguiente, no es tan claro que la especificación propuesta por Cussins sea la que debamos usar.

\section{Tercera parte: del contenido conceptual a la acción}

Para terminar, me gustaría ampliar la observación que de Vignemont y Alsmith hacen a propósito de cómo representaciones con distinto tipo de contenido mental se conectan con nuestras acciones. Esta discusión será programática, sin duda, pues un tratamiento a profundidad del tema exige abordar de manera exhaustiva temas de filosofia de la acción, asuntos como el concepto de racionalidad y la toma de decisiones, entre otros, lo cual desborda los propósitos de este artículo.

De Vignemont y Alsmith sostienen que "el papel de las representaciones en la acción no es un asunto de todo o nada... Aquí, y seguramente en otras partes, la conexión entre representación y acción es una cuestión de grado; es una cuestión de cuán directa o inmediata es la transición de la representación a la acción"43. Como ejemplo de esta afirmación, mencionan el siguiente caso:

130 "Una persona va a la cocina, y lo hace porque cree que hay chocolate allî", que consideran que evidencia de que "incluso estados altamente cognitivos de nivel personal pueden ser antecedentes causales de la acción (o al menos estar implicados explicativamente por una acción)"44.

\footnotetext{
${ }^{43}$ Alsmith, A. y F. de Vignemont, "Embodying the Mind and Representing the Body", p. 6. ${ }^{44}$ Ibid.
} 
Afirmar que distintos contenidos mentales pueden ser más o menos relevantes para la realización de acciones (puesto que el grado en el que anteceden causalmente una acción puede variar) es consistente con lo que hemos venido discutiendo a propósito del hecho de que ser general y objetivo, por un lado, y estar conectado con la acción, por otro, son propiedades opuestas del contenido mental. Para ser más precisos, en lugar de decir que son mutuamente excluyentes, podría decirse que ser general y objetivo y estar relacionado con nuestras acciones, son propiedades que se dan de manera inversamente proporcional en el contenido representacional ${ }^{45}$. En este orden de ideas, la dependencia del contexto que tiene un contenido representacional aumenta cuanto más alta sea la relación del mismo con nuestras acciones. En la medida que ser general y ser objetivo son propiedades del contenido que implican que este no es dependiente de un contexto, podemos deducir que cuanto más objetivo y general sea un contenido, menor será su relación con nuestras acciones (es decir, su relación con la acción será mediada e indirecta). Lo que hemos venido argumentando a propósito del contenido de las representaciones del cuerpo involucradas en la orientación de nuestras acciones cotidianas es que no exhibiria las propiedades de ser general y ser objetivo, en la medida en que tendría la más alta relación con nuestras acciones.

Esta perspectiva coincide con lo que ha afirmado Clark $^{46}$ en el sentido de que el contenido mental es un continuo donde, junto a las representaciones mentales clásicas (ubicadas por este autor en lo alto del continuo), se encuentra también la que él denomina "cognición biológica" centrada en la acción (y que él ubica hacia la mitad de dicho continuo ${ }^{47}$. Igualmente, coincide con lo señalado por Cussins ${ }^{48}$ con respecto a que las propiedades de ser general y ser objetivo constituyen simplemente una de las trayectorias posibles en el espacio disponible para las representaciones.

De esta manera, solo un contenido mental que tenga el mayor grado de relacionamiento con nuestras acciones estará conectado con ellas inmediatamente y directamente. Dicho de otro modo, ningún otro contenido mental sería

\footnotetext{
${ }^{45}$ La plausibilidad de esta sugerencia aumenta si tenemos en cuenta que ser general, objetivo y estar relacionado con nuestras acciones son asuntos de grado. Tomemos el caso de los contenidos demostrativos ("ese color") u otros de tipo indexical o egocéntrico ("aquí"), los cuales claramente exhiben una generalidad y objetividad menor que aquellos que se encuentran en estados cognitivos abstractos (como, digamos, las creencias verdaderas sobre topologia diferencial).

${ }^{46}$ Clark, A., Being There: Putting Brain, Body and World Together Again, Cambridge: MIT Press, 1997, p. 147.

${ }^{47}$ Ibid., p. 114.

${ }^{48}$ Cussins, A., "Content, embodiment, and objectivity: The theory of cognitive trails", p. 684.
} 
directamente explotable para actuar. Nuestro argumento ha sido que la única manera en que el contenido mental puede tener este rasgo es si es intrínsecamente motivacional para su sujeto, como defendemos que sucede en el caso del contenido mental cuyo modo de presentación son posibilidades de acción. De Vignemont y Alsmith admiten este punto cuando anotan que "en el caso de la creencia sobre chocolate de la persona, el formato no es directamente explotable para guiar su acción... No tiene dimensión motivacional..."49. Así, puesto que no son intrínsecamente motivacionales, es necesario agregar pasos intermedios para que haya una transición de las creencias y afines a la acción.

El proceso mediante el cual tiene lugar esta transición generalmente se explica mediante del concepto de razonamiento práctico. Grosso modo, en un razonamiento práctico los contenidos conceptuales de algunos estados mentales (como las actitudes proposicionales y los juicios basados en percepciones, entre otros) tienen el lugar de premisas, de las cuales se deriva una intención. Por ejemplo: María cree que debe llegar a un punto distante en la ciudad (premisa 1) y juzga que cierta ruta de autobús es la indicada para llegar a su destino (premisa 2), por lo cual forma la intención de tomar la ruta indicada. En otras palabras, la idea es que, para que puedan influir en nuestras acciones, los estados mentales que tienen contenido conceptual deben hacer parte de procesos inferenciales donde la conclusión toma la forma de una intención.

Sin embargo, recordemos de nuevo el argumento de Searle ${ }^{50}$, según la cual existe una brecha que separa las intenciones previas de la acción. De ser correcto, este argumento implicaría que la formación de intenciones no sería suficiente para dar lugar a acciones. Como mencionamos anteriormente, Searle sostiene que se puede cerrar esta brecha mediante la introducción de "intenciones-en-la-acción", que guían la acción de manera directa. Sin embargo, como también hemos subrayado, estas intenciones deben cumplir ciertas condiciones para poder cerrar la brecha (en especial, su contenido debe ser intrínsecamente motivacional) ${ }^{51}$. De lo contrario, con su introducción el problema simplemente habría sido desplazado.

El contenido que hemos propuesto para las representaciones del cuerpo involucradas en la orientación de las acciones cotidianas podria proponerse como el de las intenciones introducidas por Searle (o, al menos, parte de este).

\footnotetext{
${ }^{49}$ Alsmith, A. y F. de Vignemont, "Embodying the mind and representing the body", en: Review of Philosophy and Psychology, p. 6.

${ }^{50}$ Searle, J., Rationality in action, Cambridge: MIT press, 2001.

${ }^{51}$ Pacherie, E., "The Content of Intentions", en: Mind and Language, v. IV, 15 (2000), pp. 400-432.
} 
$\mathrm{Si}$, en efecto, debe haber intenciones-en-la-acción que guian directamente la acción, su contenido ciertamente deberá ser intrínsecamente motivacional. Como resultado, para que puedan incidir en nuestras acciones, los contenidos representacionales que no están relacionados de manera directa con la acción tendrian que estar asociados con contenidos como el que hemos propuesto para las representaciones del cuerpo involucradas en la orientación de nuestras acciones cotidianas.

\section{Conclusión}

La filosofia de la mente y la ciencia cognitiva han adoptado una explicación representacional de la cognición del cuerpo y la realización de acciones, incluso cuando se trata de la ejecución de actividades que llevamos a cabo cotidianamente. De las numerosas criticas que esta postura ha suscitado, en este artículo nos enfocamos en algunas de las presentadas por Merleau-Ponty: que representar el cuerpo es incompatible con que este sea dado como lo que hace posible la percepción y que las actividades cotidianas pueden ser explicadas por un tipo de intencionalidad distinto de la intencionalidad de acto típica de los juicios. Al respecto, argumentamos que la primera critica contiene supuestos cuestionables y que la intencionalidad motora u operante no sería incompatible con la noción de contenido representacional. No obstante, sostuvimos que esto no significa que las dos críticas debieran ser hechas a un lado, sino que resultaba necesario extraer dos intuiciones que estas contienen y que restringen cualquier explicación que se quiera dar del contenido de representaciones del cuerpo que estén involucradas en la orientación de acciones cotidianas.

La primera intuición es que el cuerpo es un objeto intencional único, que no puede ser considerado como equivalente a cualquier otro objeto que nos es dado. Así, una teoría del contenido de las representaciones del cuerpo debe reconocer estas particularidades, en especial cuando se trata de su involucramiento en la realización de actividades cotidianas. La segunda intuición es que la relación entre el contenido de estas representaciones del cuerpo y la acción debe ser evidente y directa, al contrario de lo que sucede con el contenido de estados mentales como las creencias. Como anotamos, contenidos proposicionales o conceptuales como los de las creencias no parecer ser condición necesaria ni suficiente para la realización de ciertas actividades cotidianas. En este sentido, nos adherimos a la tesis de que un contenido mental satisface la segunda intuición si es intrínsecamente motivacional. 
Si el contenido de las representaciones del cuerpo involucradas en la orientación de las acciones cotidianas no es como el contenido proposicional o conceptual de las creencias, nuestra inferencia fue que debe tratarse de un contenido mental no-conceptual orientado a la acción. Específicamente, nuestra propuesta es que se trata de un contenido cuyo modo de presentación es en términos de posibilidades de acción. Tres consecuencias de la propuesta se destacan: la primera es que la conexión directa con la acción hace que el contenido en cuestión sea altamente dependiente del contexto y, por lo tanto, no sea general y objetivo; la segunda es que, en este contenido, la distinción entre actitud y contenido no parece pertinente; la tercera es que, si bien la propuesta comparte premisas con las representaciones pushmi-pullyu de Millikan, la manera en que se especifican unas y otras es significativamente distinta.

El asunto de la especificación del contenido propuesto nos llevó a analizar el argumento de Cussins según el cual el contenido mental no-conceptual no puede tener especificaciones con condiciones de verdad. Lo que encontramos fue que la necesidad, admitida por Cussins, de distinguir entre la posesión de un contenido mental y comprender su especificación permite afirmar que una especificación con condiciones de verdad puede corresponder a un contenido mental no-conceptual. Con respecto al tipo de especificación que propone Cussins, tampoco es claro que sea necesariamente mejor que otras especificaciones para capturar el significado cognitivo del contenido mental no-conceptual.

Por último, abordamos la pregunta sobre la manera en que diversos tipos de contenido mental se relacionan con la acción. Siguiendo la observación de Alsmtith y de Vignemont según la cual esta relación es una cuestión de grado, matizamos lo afirmado previamente con respecto a que la dependencia del contexto y la relación con la acción eran propiedades del contenido mental opuestas a las de ser general y ser objetivo; se trataria de propiedades que se presentan de manera inversamente proporcional. Solo contenidos donde la dependencia del contexto y la relación con la acción sean las más altas podrían incidir de manera directa en la acción, mientras que otros tipos de contenido deberán hacer parte de razonamientos prácticos que den lugar a intenciones (las cuales, quizás, también requieran contenidos directamente explotables en la acción para poder incidir en ellas). El contenido de las representaciones del cuerpo involucradas en la orientación de acciones cotidianas seria, precisamente, un contenido del primer tipo.

La propuesta de contenido de estas representaciones del cuerpo que hemos delineado, sin duda, debe complementarse con una descripción de los 
aspectos del cuerpo que este contenido incluye (por ejemplo, si se trata únicamente de propiedades especiales). También resultaría pertinente establecer la manera en que esta propuesta se conecta con la forma en la que se ven las representaciones del cuerpo en las ciencias cognitivas y, quizás, si puede contribuir a la elucidación de diversos asuntos de la cognición del cuerpo (como la experiencia de nuestro cuerpo como propio, la localización de sensaciones corporales, etcétera). Estos caminos de investigación futura apuntan, pues, a ampliar la propuesta y determinar su lugar en el debate más amplio sobre la manera en que sabemos de nuestro cuerpo.

Recibido: 19/09/2017

Aceptado: 04/07/2019

\section{Bibliografia}

Alsmith, A. y F. de Vignemont, "Embodying the mind and representing the body", en: Review of Philosophy and Psychology, v. I, 3 (2012), pp. 1-13. https://doi. org/10.1007/s13164-012-0085-4

Arias, B., "La intencionalidad operante en Maurice Merleau-Ponty", en: Contrastes: revista internacional de filosofia, II (1997), pp. 5-25.

Blanke, O. y Mohr, C., "Out-of-body experience, heautoscopy, and autoscopic hallucination of neurological origin: Implications for neurocognitive mechanisms of corporeal awareness and self-consciousness", en: Brain Research Reviews, V. I, 50 (2005), pp. 184-199. https://doi.org/10.1016/j.brainresrev.2005.05.008

Brewer, T., "Three Dogmas of Desire", en: Values and Virtues: Aristotelianism in Contemporary Ethics, Oxford University Press (2006), pp. 257-284.

Clark, A., Being There: Putting Brain, Body and World Together Again, Cambridge: MIT Press, 1997. https://doi.org/10.7551/mitpress/1552.001.0001

Crane, T., "Conceptual and Non-conceptual Content", en: The Routledge Encyclopedia of Philosophy, Taylor and Francis (1998).

Cussins, A., "Content, Embodiment, and Objectivity: The Theory of Cognitive Trails", en: Mind, v. CDIV, 101 (1992), pp. 651-688. https://doi.org/10.1093/ mind/101.404.651

Cussins, A., "Content, Conceptual Content, and Nonconceptual Content", en: Essays on Nonconceptual Content, MIT Press (2002), pp. 133-163.

Cussins, A., "Environmental Representation of the Body", en: Review of Philosophy and Psychology, v. III, 1 (2012) pp. 15-32. https://doi.org/10.1007/s13164-012-0086-3

De Vignemont, F., "Body Schema and Body image-Pros and Cons", en: Neuropsychologia, v. XLVIII, 3 (2010), pp. 669-680. https://doi.org/10.1016/j. neuropsychologia.2009.09.022 
Dreyfus, H., "Intelligence without Representation - Merleau-ponty's Critique of Mental Representation the Relevance of Phenomenology to Scientific Explanation", en: Phenomenology and the Cognitive Sciences, v. IV, 1 (2002), pp. 367-383. https:// doi.org/10.1023/A:1021351606209

Gallagher, S., How the Body Shapes the Mind, Nueva York: Oxford University Press, 2005. https://doi.org/10.1093/0199271941.001.0001

Gibson, J., The Ecological Approach to Visual Perception, Boston: Houghton Mifflin, 1979.

Lycan, W., "Desire Considered as a Propositional Attitude", en: Philosophical Perspectives, v. XXVI, 1 (2012), pp. 201-215. https://doi.org/10.1111/phpe.12003

Merleau-Ponty, M., Phénoménologie de la Perception, Paris: Gallimard, 1945.

Millikan, R., "Pushmi-pullyu Representations", en: Philosophical Perspectives, IX (1995), pp. 185-200. https://doi.org/10.2307/2214217

O’Shaughnessy, B., The Will (Vol. I), Cambridge: Cambridge University Press, 1980.

O'Shaughnessy, B., "Proprioception and the Body Image", en: The Body and the Self, MIT Press (1995), pp. 175-203.

Oppy, G., "PropositionalAattitudes”, en: The Routledge Encyclopedia of Philosophy, Taylor and Francis (1998).

Pacherie, E., "The Content of Intentions", en: Mind and Language, v. IV, 15 (2000), pp. 400-432. https://doi.org/10.1111/1468-0017.00142

Peacocke, C., "Scenarios, Concepts, and Perception", en: The Contents of Experience, Cambridge University Press (1992), pp. 105-135. https://doi.org/10.1017/ CBO9780511554582.006

Searle, J., Rationality in Zction, Cambridge: MIT press, 2001. https://doi. org/10.1080/10002001058538709

Strawson, G., Mental Reality. Cambridge: MIT Press, 1994.

Thagard, P., "Desires are not Propositional Attitudes", en: Dialogue, v. I, 45 (2006), pp. 151-156. https://doi.org/10.1017/S001221730000038X

Wheeler, M., Reconstructing the Cognitive World: The Next Step, Cambridge: MIT Press, 2005. https://doi.org/10.7551/mitpress/5824.001.0001 